\title{
The Minimum Temperature for Levitating Droplets
}

\author{
For water on hot surfaces, the Leidenfrost effect endures at temperatures \\ much lower than those needed for onset, regardless of surface or fluid \\ properties.
}

By Christopher Crockett

$\square$ lace a water droplet on a hot enough surface, and it will levitate on a cushion of water vapor. This "Leidenfrost effect" has been known about since 1756 , and yet, reported values of the precise temperature at which the vapor forms vary widely. Now, a new study shows that, for water, the vapor layer endures at temperatures much lower than those required for its formation, independent of the water's salinity, the vapor volume, or the type of heated material [1].

Leidenfrost experiments with actual levitating droplets introduce many hard-to-control variables. So Dana Harvey and colleagues at Emory University in Georgia took a new approach: They dunked a heated metal cylinder with a rounded tip into a water bath and monitored the electrical impedance between the cylinder and an electrode at the bottom of the bath. When a vapor layer formed beneath the tip, it introduced a capacitance that varied with vapor thickness. By changing the cylinder's temperature, the team could then pinpoint the onset of vapor

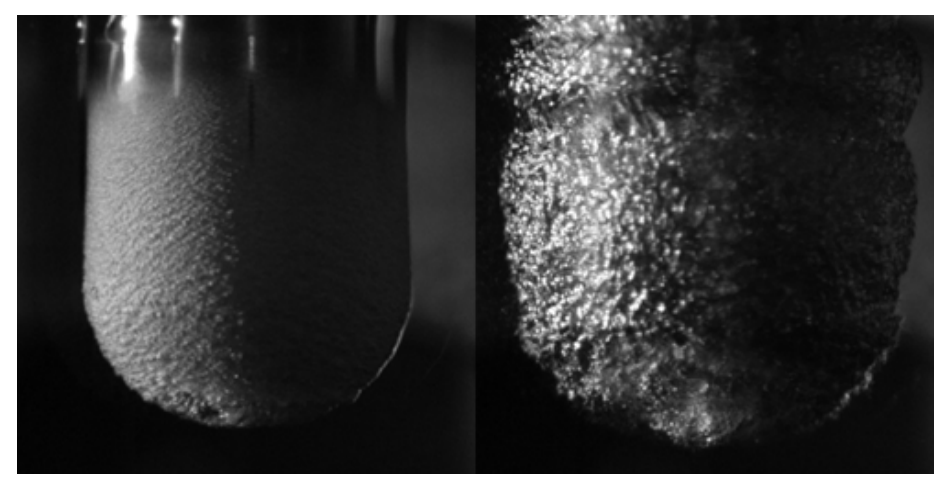

Credit: D. Harvey, J. Méndez Harper, J. C. Burton/Emory University formation.

On average, a stable vapor layer formed at around $240^{\circ} \mathrm{C}$, with the precise temperature varying based on the type of metal used for the cylinder. But regardless of metal type or water salinity, the vapor persisted until the temperature dropped to $140^{\circ} \mathrm{C}$, triggering a vapor collapse that was "explosive and audible." This consistency suggests that the minimum temperature is set by the stability of gas flow within the vapor layer rather than the properties of the water or the heated surface, the team says. What exactly triggers the collapse remains an open question.

Christopher Crockett is a freelance writer based in Arlington, Virginia.

\section{REFERENCES}

1. D. Harvey et al., "Minimum Leidenfrost temperature on smooth surfaces," Phys. Rev. Lett. 127, 104501 (2021). 